\title{
Anti-Hyperglycemic Properties of Crude Extract and Triterpenes from Poria cocos
}

\author{
Tzu-Hsuan Li, ${ }^{1,2}$ Chia-Chung Hou, ${ }^{1}$ Cicero Lee-Tian Chang, ${ }^{3}$ and Wen-Chin Yang ${ }^{1,2,4,5}$ \\ ${ }^{1}$ Agricultural Biotechnology Research Center, Academia Sinica Taipei 115, Taiwan \\ ${ }^{2}$ Department and Institute of Pharmacology, National Yang-Ming University Taipei 112, Taiwan \\ ${ }^{3}$ Department of Veterinary Medicine, National Chung Hsing University Taichung, Taiwan \\ ${ }^{4}$ Institute of Zoology, National Taiwan University Taipei 10617, Taiwan \\ ${ }^{5}$ Department of Life Sciences, National Chung Hsing University Taichung, Taiwan
}

Correspondence should be addressed to Cicero Lee-Tian Chang, ltchang@nchu.edu.tw and Wen-Chin Yang, wcyang@gate.sinica.edu.tw

Received 1 April 2010; Accepted 7 August 2010

Copyright (๑) 2011 Tzu-Hsuan Li et al. This is an open access article distributed under the Creative Commons Attribution License, which permits unrestricted use, distribution, and reproduction in any medium, provided the original work is properly cited.

Poria cocos, Bai Fu Ling in Chinese, is used in traditional Chinese medicine to treat diabetes. However, its claimed benefits and mechanism are not fully understood. This study aimed to investigate the effect and action of $P$. cocos on type 2 diabetes. We first performed phytochemical analysis on the crude extract and factions of $P$. cocos. $P$. cocos crude extract at $50 \mathrm{mg} / \mathrm{kg}$ body weight or more significantly decreased blood glucose levels in $\mathrm{db} / \mathrm{db}$ mice. Based on a bioactivity-directed fractionation and isolation (BDFI) strategy, chloroform fraction and subfractions 4 and 6 of the P. cocos crude extract possessed a blood glucose-lowering effect. Dehydrotumulosic acid, dehydrotrametenolic acid, and pachymic acid were identified from the chloroform sub-fractions 4, 3, and 2, respectively. Dehydrotumulosic acid had anti-hyperglycemic effect to a greater extent than dehydrotrametenolic acid and pachymic acid. Mechanistic study on streptozocin- (STZ-) treated mice showed that the crude extract, dehydrotumulosic acid, dehydrotrametenolic acid, and pachymic acid of $P$. cocos exhibited different levels of insulin sensitizer activity. However, the $P$. cocos crude extract and triterpenes appeared not to activate PPAR- $\gamma$ pathway. Overall, the data suggest that the P. cocos extract and its triterpenes reduce postprandial blood glucose levels in $\mathrm{db} / \mathrm{db}$ mice via enhanced insulin sensitivity irrespective of PPAR- $\gamma$.

\section{Introduction}

Diabetes mellitus is a life-threatening chronic metabolic disease which currently afflicts $3 \%$ of the world population. Over $90 \%$ of diabetic populations are diagnosed with type 2 diabetes $[1,2]$. Diabetes is caused by a defect in insulin production, insulin action, or both [3]. The defect impairs glucose homeostasis in diabetic patients, resulting in hyperglycemia, a hallmark of diabetes [4]. Therefore, one important method of treating patients with type 2 diabetes is to control blood glucose levels, which can be achieved by an increase of insulin release (insulin releasers) or insulin action (insulin sensitizers), a decrease of intestinal glucose uptake ( $\alpha$-glucosidase inhibitors), and so forth [5]. However, few clinical drugs are available for diabetes, and those that are available usually have adverse side effects such as decreased efficacy over time and low cost-effectiveness [6$8]$. Therefore, research and development of novel drugs for diabetes have been in great demand.
P. $\operatorname{cocos}$ (Polyporaceae) is a rotten pine-tree fungus. It has long been used as traditional Chinese medicine and food [9-12]. P. cocos alone or in combination with other herbs is often used to treat diabetes as well as other disorders [13-15]. Several triterpenes, pachyman, and pachymaran have been identified from $P$. cocos [16-19]. Dehydrotrametenolic acid, one triterpene constituent of $P$. cocos, was shown to reduce hyperglycemia in $\mathrm{db} / \mathrm{db}$ mice [20]. Besides, dehydrotrametenolic acid was shown to activate peroxide proliferator-activated receptor- $\gamma$ (PPAR- $\gamma$ ), a regulator of glucose metabolism, in the same way as the insulin sensitizer ciglitazone [20]. However, this antidiabetic effect and the likely mechanism of $P$. cocos are still poorly studied.

In this study, we used $\mathrm{db} / \mathrm{db}$ mice to evaluate the glucose-lowering effects of the crude extract, fractions, and triterpenes of $P$. cocos. Based on a bioactivity-directed fractionation and isolation (BDFI) procedure, triterpenes from the crude extract of $P$. cocos were identified as active compounds. Chromatography and spectroscopy were used 
to characterize the phytochemistry of $P$. cocos. In addition, STZ-treated mice were used to study the likely mechanism of P. cocos and its active triterpenes.

\section{Materials and Methods}

2.1. Chemicals, Cells, and Plasmids. Dimethyl sulfoxide (DMSO), glimepiride (GLM), metformin (Met), methanol, ethyl acetate (EA), chloroform, acetonitrile (ACN), trifluoroacetic acid (TFA), and streptozocin (STZ) were purchased from Sigma-Aldrich (MO, USA). Pachymic acid and insulin were purchased from Apin Chemicals Limited (Oxon, UK) and Novo Nordisk (NJ, USA), respectively. 3T3-L1 adipocytes were grown in DMEM medium (Invitrogen, CA, USA) supplemented with $10 \%$ bovine calf serum (BCS), 100 units $/ \mathrm{mL}$ penicillin, $100 \mu \mathrm{g} / \mathrm{mL}$ streptomycin, $6 \mathrm{mM}$ HEPES (Invitrogen, CA, USA), and $2 \mathrm{mM}$ L-glutamine (Invitrogen) at $37^{\circ} \mathrm{C}$ in $5 \% \mathrm{CO}_{2}$. pSGGAL-PPAR- $\gamma$, which expresses a chimeric protein of Gal4 DNA-binding domain linked to PPAR- $\gamma$ ligand-binding domain, and (UAS)5-tkLUC reporter construct, which contains a pentameric yeast upstream activatory sequence linked to luciferase gene, were generous gifts from Dr. Krister Bamberg (Astra Zeneca, Mölndal, Sweden).

2.2. Preparation of Crude Extract, Fractions, and Triterpenes of $P$. cocos. $P$. cocos was purchased from a local market in Taipei, Taiwan. It was authenticated by the Development Center for Biotechnology, Taiwan. Voucher specimens were deposited at Agricultural Biotechnology Research Center. To prepare methanol crude extract of $P$. cocos, dried $P$. cocos was ground into powder. $P$. cocos powder $(5 \mathrm{~kg})$ was extracted with methanol $(1: 10 \mathrm{w} / \mathrm{v})$ for 6 days three-times, yielding $7.5 \%$ methanol extract. The methanol crude extract was dissolved in water $(1 \mathrm{~L})$ and partitioned with chloroform $(1 \mathrm{~L} \times 5)$. After obtaining the chloroform fraction $(0.82 \%$ ), the rest of the extract was further partitioned with ethyl acetate $(1 \mathrm{~L} \times 5)$, yielding an ethyl acetate fraction $(2.9 \%$ ) and a water fraction $(1.8 \%$ ). The active chloroform fraction was further fractioned into chloroform sub-fractions $1(0.10 \%)$, 2 (0.11\%o), 3 (0.11\%o), 4 (0.12\%o), 5 (0.10\%o), and 6 $(0.24 \%$ ) using a normal phase high-performance liquid chromatography (HPLC) column (Phenomenex, Luna $5 \mu$ silica, $250 \times 10 \mathrm{~mm}$ ) at a flow rate of $5 \mathrm{ml} / \mathrm{min}$, detected with UV $242 \mathrm{~nm}$. The solvent gradient for HPLC is ethyl acetate (a) and hexane (b): 0 to $60 \mathrm{~min}$ : $30 \% \mathrm{~A}$; 60 to $90 \mathrm{~min}$ : $100 \%$ A. The 6 chloroform sub-fractions were chemically characterized as published elsewhere [21] using an RP-C18 HPLC column (Phenomenex, Luna $5 \mu \mathrm{C} 18,250 \times 4.6 \mathrm{~mm}$ ) at a flow rate of $1 \mathrm{ml} / \mathrm{min}$, detected with UV $242 \mathrm{~nm}$. The solvent gradient for HPLC is water/0.05\% TFA (c) and acetonitrile/0.05\% TFA (d): 0 to $15 \mathrm{~min}: 5$ to $15 \%$ D; 15 to $35 \mathrm{~min}$ : 15 to $20 \% \mathrm{D}$; 35 to $65 \mathrm{~min}$ : 20 to $40 \%$ D; 65 to $100 \mathrm{~min}$ : 40 to $100 \% \mathrm{D} ; 100$ to $110 \mathrm{~min}: 100 \%$ D. Triterpenes were identified from the chloroform subfractions using an RP HPLC column (Cosmosil, $5 \mu \mathrm{C} 18$, $250 \times 10 \mathrm{~mm}$ ) at a flow rate of $5 \mathrm{ml} / \mathrm{min}$, detected with UV $242 \mathrm{~nm}$. The solvent gradient for HPLC was water $/ 0.05 \%$
TFA (e) and acetonitrile $/ 0.05 \%$ TFA (f): 0 to $10 \mathrm{~min}: 30$ to $52 \% \mathrm{~F}$; 10 to $50 \mathrm{~min}$ : 52 to $100 \% \mathrm{~F}$; 50 to $60 \mathrm{~min}$ : $100 \%$ F. Dehydrotumulosic acid, dehydrotrametenolic acid, and pachymic acid were identified by comparing the data obtained from NMR and MS with those published previously in [21-23].

\subsection{Animal and Drug Administration. Male C57BL/KsJ-} $\mathrm{db} / \mathrm{db}$ mice, which possess a point mutation of the leptin receptor, and male $\mathrm{C} 57 \mathrm{BL} / 6 \mathrm{~J}$ mice were purchased from the Jackson Laboratory (ME, USA) and the National Laboratory Animal Center (Taipei, Taiwan), respectively. All animals were housed and handled according to the guidelines of the Academia Sinica Institutional Animal Care and Utilization Committee.

Diabetic C57BL/KsJ-db/db mice aged between 6 to 8 weeks were denied food for $12 \mathrm{~h}$ before the experiment and then given free access to food and water for $2 \mathrm{~h}$. On time 0 , food was removed (water accessible) and blood sampling from this time point on was considered to be postprandial. After grouping, mice were tube-fed with vehicle, metformin, crude extract, fractions, sub-fractions, or compounds. Postprandial blood glucose level was monitored for $4 \mathrm{~h}$.

C57BL/6J mice aged 5 weeks were treated with an intraperitoneal injection of STZ $(200 \mathrm{mg} / \mathrm{kg})$. Mice with postprandial blood glucose level over $500 \mathrm{mg} / \mathrm{dL}$ and serum insulin level below $0.18 \mathrm{ng} / \mathrm{ml}$ were grouped and tube-fed with control, metformin, glimepiride, crude extract, and compounds (time-1 h). Sixty minutes after tube-feeding of mice, the mice were intraperitoneal injected with insulin at $2.5 \mathrm{IU} / \mathrm{kg}$ BW (time 0). Blood glucose levels were measured for $4 \mathrm{~h}$, and all the time points were given free access to food and water. Blood glucose concentration was measured using an Elite glucometer (Bayer, PA, USA). This experiment was modified by previous studies $[24,25]$.

2.4. PPAR- $\gamma$ Reporter Assay. 3T3-L1 $\left(10^{7}\right)$ cells were electroporated with pSGGAL-PPAR- $\gamma$ and (UAS)5-tk-LUC at $260 \mathrm{~V}$ and $975 \mu \mathrm{F}$ using a Bio-Rad electroporator (BioRad, Hercules, CA). Two hours after transfection, cells were divided and treated with DMSO, rosiglitazone (RSG), the crude extract, and triterpenes of $P$. cocos for $24 \mathrm{~h}$. Ten $\mu \mathrm{g}$ of total lysates underwent dual luciferase assays as published in [26]. PPAR- $\gamma$ activity in folds was defined as the firefly/Renilla luciferase ratio normalized to the control firefly/Renilla luciferase ratio.

2.5. Statistical Analysis. The results from three or more independent experiments were presented as mean \pm S.E. Data were analyzed by ANOVA. Bonferroni's method was used for post hoc comparisons when appropriate to determine the source of significant differences. Differences of $P$-value less than .05 were considered statistically significant.

\section{Results}

3.1. Phytochemical Analysis of Crude Extract, Fractions, SubFractions, and Triterpenes of P. cocos. P. cocos has been used 


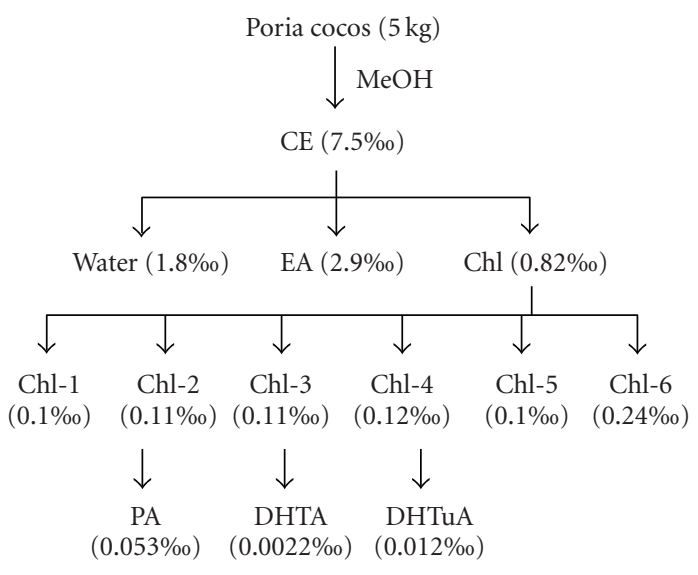

(a)

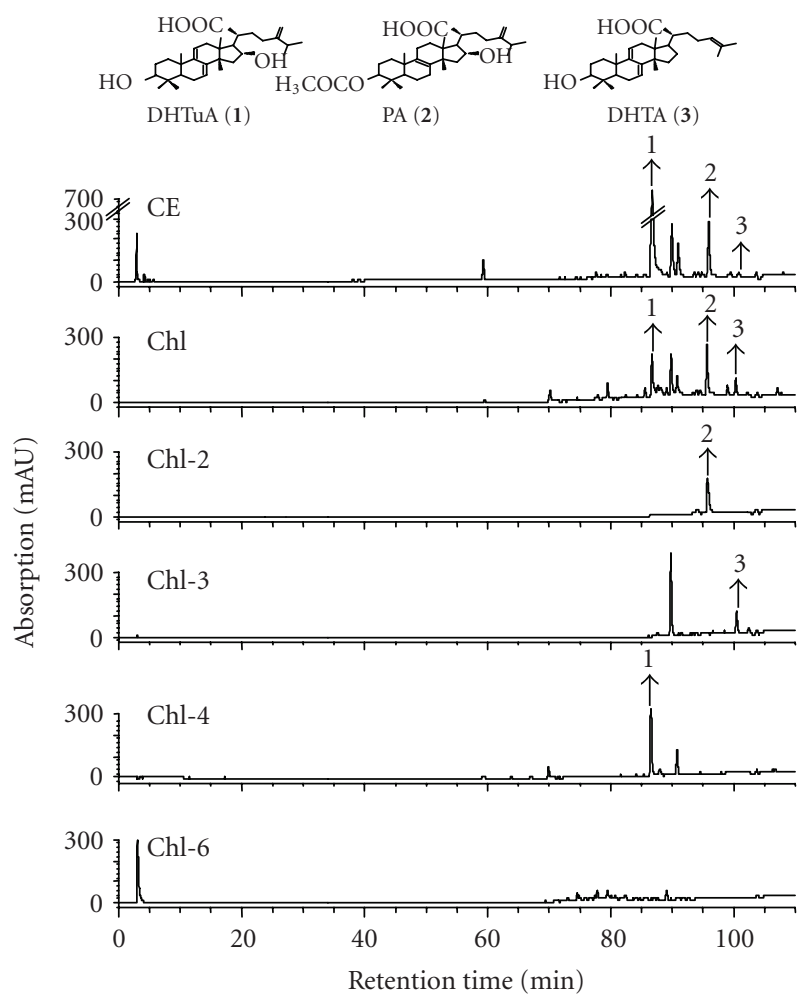

(b)

Figure 1: Isolation procedure and HPLC profiles of crude extract, fractions, sub-fractions, and triterpenes of $P$. cocos. (a) Flow chart of preparation procedure of the crude extract, fractions, sub-fractions, and triterpenes of $P$. cocos. The yield of extract, fractions, sub-fractions, and triterpenes is indicated in parentheses. (b) Chemical profiles of crude extract and fractions of $P$. cocos were obtained using HPLC columns and detected with UV detectors. Dehydrotumulosic acid (DHTuA: 1), pachymic acid (PA: 2), and dehydrotrametenolic acid (DHTA: 3 ) were identified from the Chl-4, Chl-2, and Chl-3 sub-fractions.

per se or together with other herbs as Chinese medicines for diabetes [13]. However, its anti-diabetic effect is poorly understood. In this study, we first chemically characterized crude extract, fractions, and triterpenes of $P$. cocos based on a BDFI procedure (Figure $1(\mathrm{a})$ ). To prepare the crude extract of $P$. cocos, its sclerotia were ground and extracted by methanol. The methanol crude extract was further partitioned with water, ethyl acetate, and chloroform. The active chloroform fraction was separated into 6 sub-fractions. The chloroform sub-fractions 4 and 6 were active and one triterpenes, dehydrotumulosic acid, was identified from the active chloroform sub-fraction 4. Another two triterpenes, pachymic acid and dehydrotrametenolic acid, were isolated from the inactive chloroform sub-fractions 2 and 3. The yield of the crude extract, fractions, sub-fractions, and triterpenes of $P$. cocos is indicated in Figure 1(a). Their HPLC profiles were determined as shown in Figure 1(b).

3.2. Glucose-Lowering Effects of Crude Extract, Fractions, Chloroform Sub-Fractions, and Triterpenes of P. cocos in $\mathrm{db} / \mathrm{db}$ Mice. Next, a mouse model of type 2 diabetes, $\mathrm{db} / \mathrm{db}$ mice, was used to evaluate the anti-diabetic potential of $P$. cocos. First, we examined the glucose-lowering effect of crude extract of $P$. cocos in $\mathrm{db} / \mathrm{db}$ mice. Diabetic $\mathrm{db} / \mathrm{db}$ mice aged 6 to 8 weeks spontaneously developed hyperglycemia, and their postprandial blood glucose levels were somewhere between 350 and $400 \mathrm{mg} / \mathrm{dL}$ (Figure 2(a)). In contrast, the levels of blood glucose in $\mathrm{db} / \mathrm{db}$ mice receiving a dose of control vehicle dropped to $165 \mathrm{mg} / \mathrm{dL}$ over $4 \mathrm{~h}$ (Figure 2(a)). As expected, metformin significantly lowered the levels of blood glucose (Figure $2(\mathrm{a})$ ) in $\mathrm{db} / \mathrm{db}$ mice. Crude extract of P. cocos at a single dose of 50 or $100 \mathrm{mg} / \mathrm{kg}$ body weight also significantly reduced the blood glucose levels (Figure 2(a)).

Next, we tested the glucose-lowering effect of the water, ethyl acetate, and chloroform fractions of $P$. cocos on $\mathrm{db} / \mathrm{db}$ mice. The levels of blood glucose in mice receiving a dose of control vehicle decreased to $164.5 \mathrm{mg} / \mathrm{dL}$ over $4 \mathrm{~h}$ (Figure 2(b)). Metformin effectively decreased the blood glucose levels (Figure 2(b)). In contrast, the chloroform fraction, but not the water and ethyl acetate fractions, significantly lowered blood glucose levels (Figure 2(b)). Next, we further evaluated the glucose-lowering effect of chloroform sub-fractions 1-6, resulting from the active $P$. cocos chloroform fraction. One dose of metformin effectively decreased the blood glucose levels in $\mathrm{db} / \mathrm{db}$ mice as compared to control vehicle. Chloroform sub-fractions 4 and 6 significantly lowered the blood glucose levels to a greater extent than the other 4 sub-fractions (Figure 2(c)). 


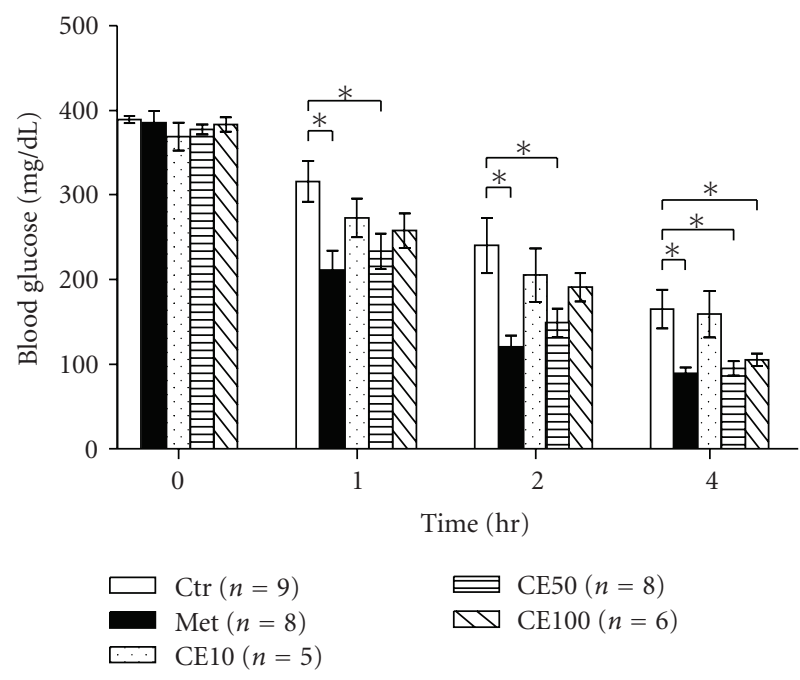

(a)

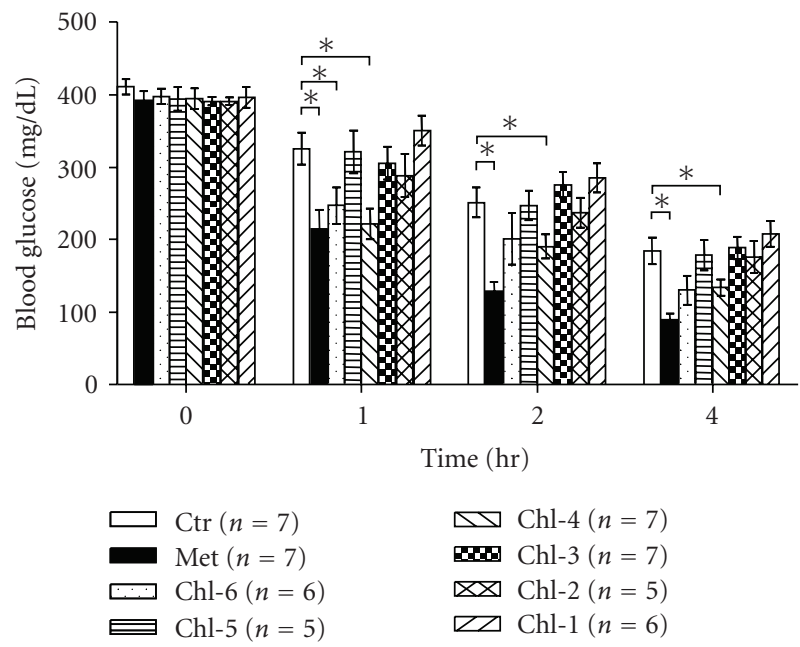

(c)

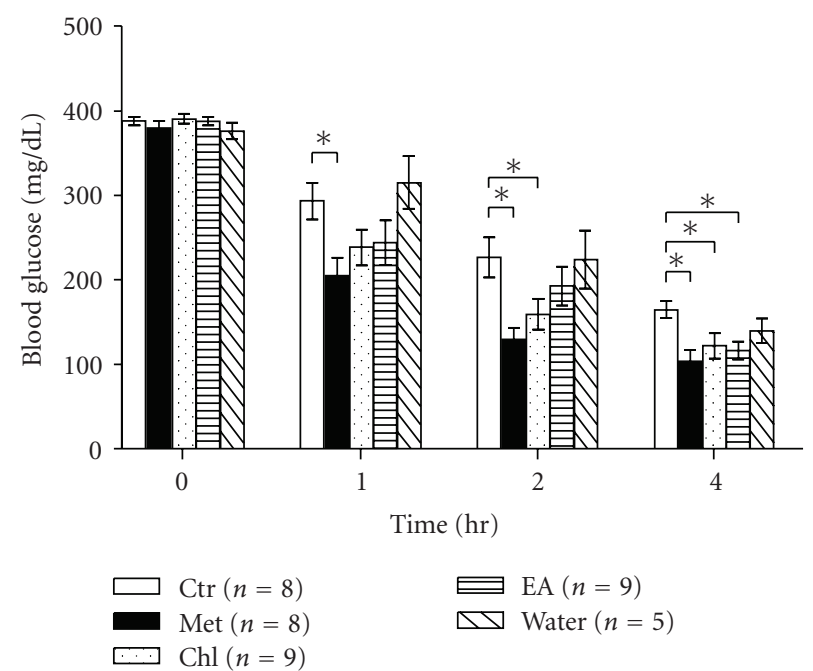

(b)
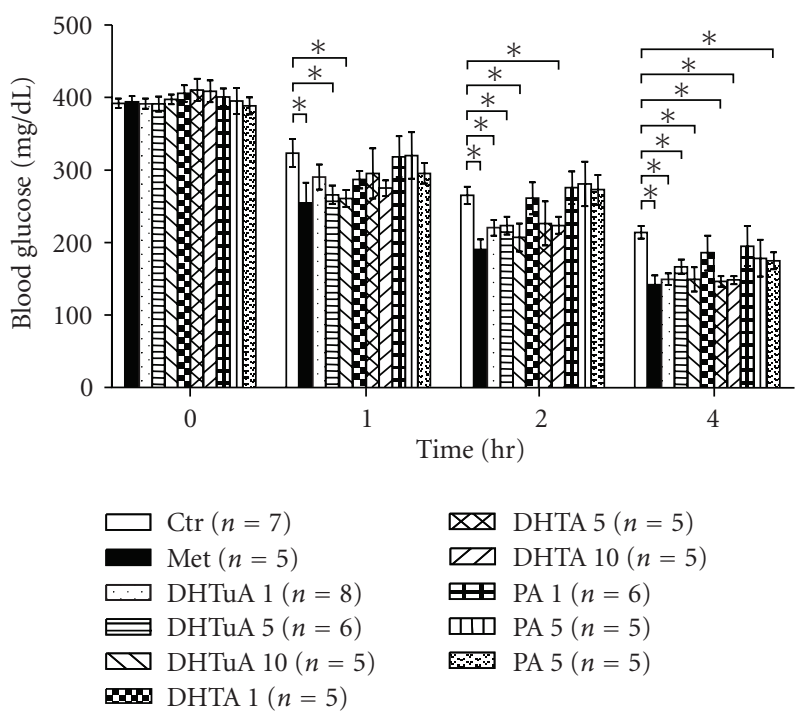

(d)

FIgURE 2: Single-dose effects of crude extract, fractions, sub-fractions, and triterpenes of $P$. cocos on the level of postprandial blood glucose in $\mathrm{db} / \mathrm{db}$ mice. (a) Diabetic db/db mice were tube-fed with water (Ctr), metformin (Met: $60 \mathrm{mg} / \mathrm{kg}$ BW), and crude extract (CE: 10, 50 or $100 \mathrm{mg} / \mathrm{kg} \mathrm{BW})$. Blood glucose levels were measured before $(0 \mathrm{~h})$ and after $(1$ to $4 \mathrm{~h})$ tube feeding. (b) The same procedure as (a) except that water (Ctr), metformin (Met: $60 \mathrm{mg} / \mathrm{kg} \mathrm{BW),} \mathrm{water} \mathrm{fraction} \mathrm{(Water:} 50 \mathrm{mg} / \mathrm{kg} \mathrm{BW}$ ), ethyl acetate fraction (EA: $50 \mathrm{mg} / \mathrm{kg}$ BW), and chloroform fraction (Chl, $50 \mathrm{mg} / \mathrm{kg} \mathrm{BW}$ ) were administered. (c) The same procedure as (a) except that water (Ctr), metformin (Met: $60 \mathrm{mg} / \mathrm{kg} \mathrm{BW}$ ), and 6 chloroform sub-fractions (Chl-1 to Chl-6: $50 \mathrm{mg} / \mathrm{kg} \mathrm{BW)} \mathrm{were} \mathrm{administered.} \mathrm{(d),} \mathrm{The} \mathrm{same} \mathrm{procedure} \mathrm{as} \mathrm{(a)} \mathrm{except}$ that DMSO/water (Ctr), metformin (Met: $60 \mathrm{mg} / \mathrm{kg}$ ), dehydrotumulosic acid (DHTuA: 1, 5, and $10 \mathrm{mg} / \mathrm{kg} \mathrm{BW),} \mathrm{pachymic} \mathrm{acid} \mathrm{(PA:} \mathrm{1,} \mathrm{5,}$ and $10 \mathrm{mg} / \mathrm{kg} \mathrm{BW}$ ), and dehydrotrametenolic acid (DHTA: 1, 5, and $10 \mathrm{mg} / \mathrm{kg} \mathrm{BW)} \mathrm{were} \mathrm{administered.} \mathrm{All} \mathrm{the} \mathrm{above} \mathrm{data} \mathrm{are} \mathrm{expressed} \mathrm{as}$ mean \pm S.E. $P ;\left(^{*}\right)$ less than .05 is considered to be statistically significant. The mouse number $(n)$ is indicated in parenthesis.

This lowering effect of sub-fraction 4 ( $50 \mathrm{mg} / \mathrm{kg}$ body weight) seemed to last longer than that of sub-fraction $6(50 \mathrm{mg} / \mathrm{kg}$ body weight) (Figure 2(c)).

Finally, we evaluated the glucose-lowering effects of triterpenes in $\mathrm{db} / \mathrm{db}$ mice. Like metformin, dehydrotumulosic acid showed a significant glucose-lowering activity in $\mathrm{db} / \mathrm{db}$ mice (Figure 2(d)). In contrast, dehydrotrametenolic acid and pachymic acid, isolated from the less active chloroform sub-fraction 3 and 2, showed lower glucose-lowering activities. However, we failed to identify active compounds from the active chloroform sub-fraction 6 of $P$. cocos.

3.3. Mechanistic Study of the Crude Extract and Triterpenes of $P$. cocos. Above we have shown that like metformin, $P$. cocos and its triterpenes have different hypoglycemic effects in $\mathrm{db} / \mathrm{db}$ mice. To understand the anti-diabetic mechanism of the crude extract of $P$. cocos and triterpenes, we examined 


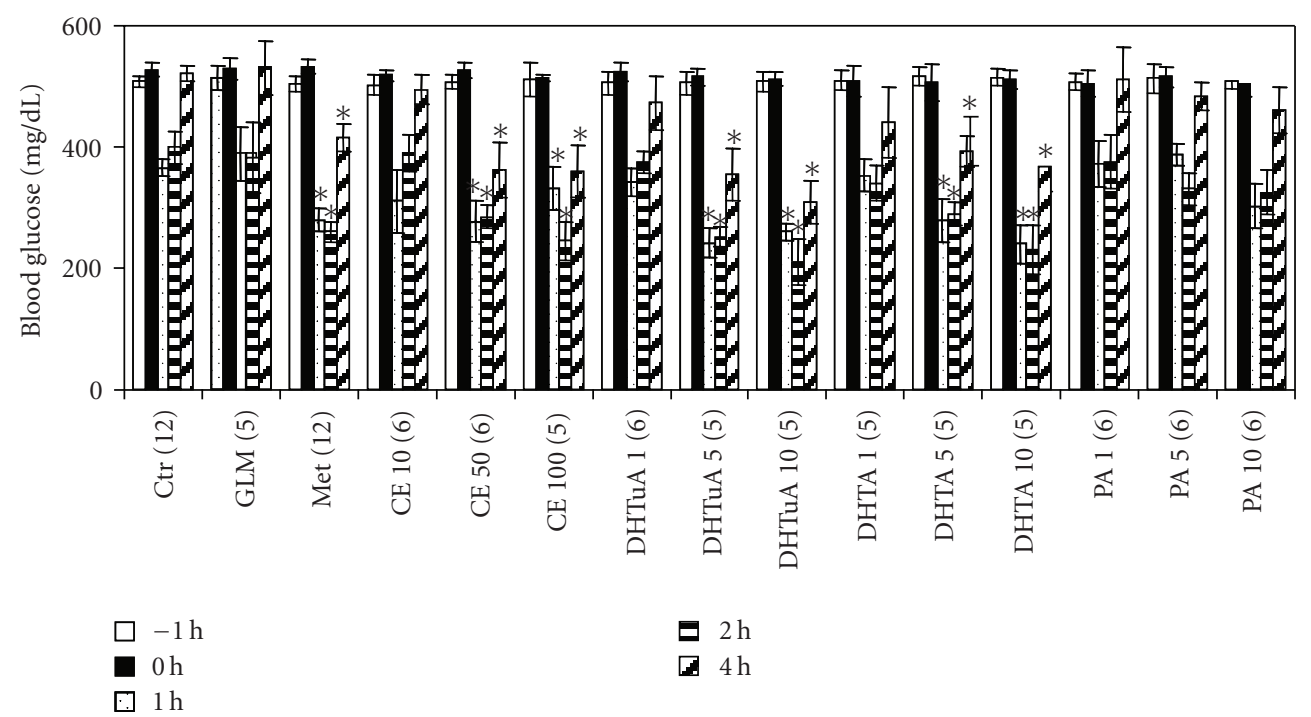

FIGURE 3: Glucose-lowering effects of the crude extract of $P$. cocos and triterpenes in STZ-treated mice. C57BL mice, which have received a high dose of STZ, were tube-fed with water (Ctr), glimepiride (GLM: $2.5 \mathrm{mg} / \mathrm{kg} \mathrm{BW}$ ), metformin (Met: $60 \mathrm{mg} / \mathrm{kg} \mathrm{BW),} \mathrm{crude} \mathrm{extract} \mathrm{(CE:}$ 10, 50, or $100 \mathrm{mg} / \mathrm{kg} \mathrm{BW}$ ), dehydrotumulosic acid (DHTuA: 1, 5, or $10 \mathrm{mg} / \mathrm{kg} \mathrm{BW}$ ), dehydrotrametenolic acid (DHTA: 1, 5, and $10 \mathrm{mg} / \mathrm{kg}$ BW), and pachymic acid (PA: 1, 5, or $10 \mathrm{mg} / \mathrm{kg}$ ). Blood glucose levels were measured before ( -1 and $0 \mathrm{~h})$ and after $(1 \mathrm{to} 4 \mathrm{~h})$ tube feeding and injection of insulin $2.5 \mathrm{IU} / \mathrm{kg}$ BW. All the above data are expressed as mean \pm S.E. $P ;\left(^{*}\right)$ indicates the difference between control (Ctr) and experimental group. The mouse number $(n)$ is indicated in parenthesis.

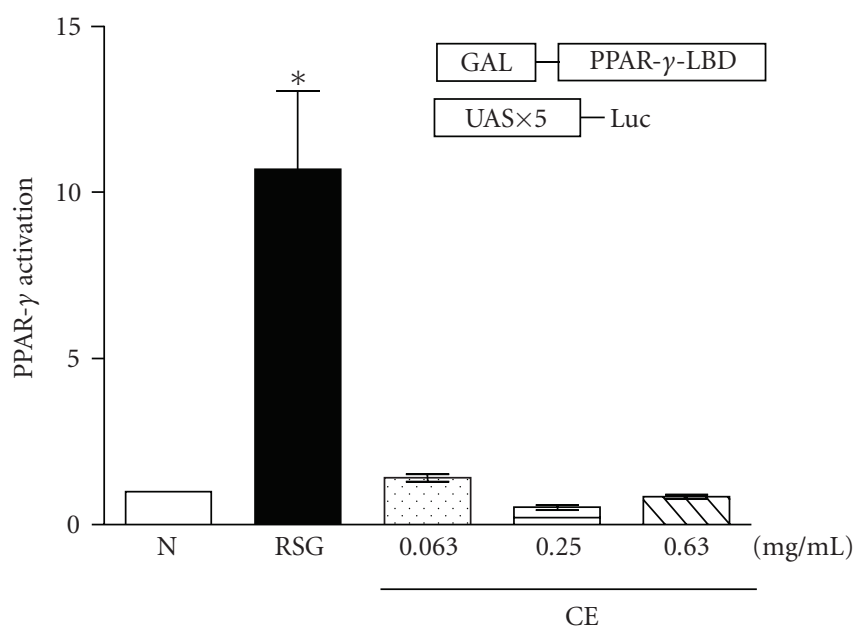

(a)

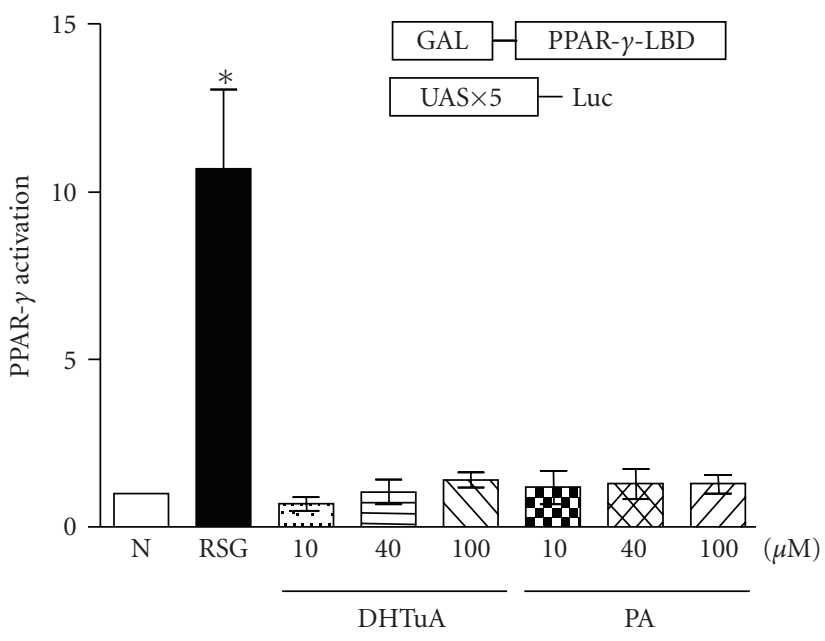

(b)

FIgure 4: Effects of the P. cocos crude extract, dehydrotumulosic acid (DHTuA), and pachymic acid (PA) on PPAR- $\gamma$ activation. (a) 3T3-L1 cells were transiently transfected with reporter constructs, pSGGAL4-PPAR- $\gamma(2 \mu \mathrm{g})$, and (UAS)5-tk-LUC plasmid (10 $\mu \mathrm{g})$. The cells were treated with DMSO (N: negative control), rosiglitazone (RSG: $1 \mu \mathrm{M}$ ), and the P. cocos crude extract $(0.063,0.25$, and $0.63 \mathrm{mg} / \mathrm{ml}$ ). After $24 \mathrm{~h}$, cell lysates were subjected to dual luciferase assay. (b) The same procedure as (a) except that the cells were treated with dehydrotumulosic acid (DHTuA: 10, 40, or $100 \mu \mathrm{M}$ ) and pachymic acid (PA: 10, 40, or $100 \mu \mathrm{M})$. All the data in folds are expressed as mean \pm S.E. P; $(*)$ less than .05 is considered to be statistically significant.

their glucose-lowering effects in STZ-treated mice whose $\beta$ islet cells were destroyed. Insulin administration was able to decrease blood glucose levels in STZ mice (Figure 3). As expected, glimepiride, an insulin releaser, had no effect on insulin-mediated blood glucose reduction in STZ-treated mice (Figure 3). In contrast, metformin, an insulin sensitizer, effectively augmented the insulin-mediated blood glucose reduction (Figure 3 ). In the same way as metformin, the crude extract of $P$. cocos also augmented the insulin-mediated blood glucose reduction (Figure 3). Similar results were obtained for dehydrotumulosic acid, dehydrotrametenolic acid and, if any, pachymic acid (Figure 3). These results imply 

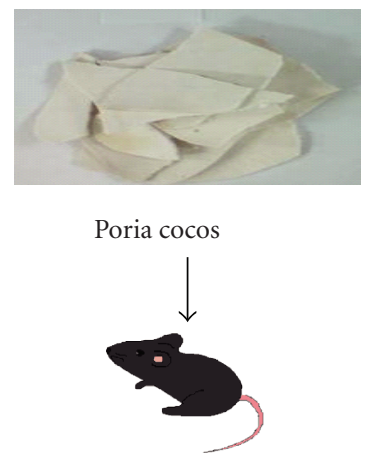

$\mathrm{db} / \mathrm{db}$ mouse model BDFT $\downarrow$ Glocose-lowering activity

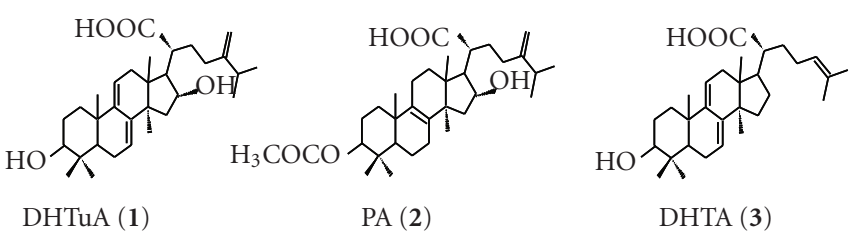

Active compounds

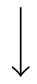

Anti-diabetic mechanism via enhanced insulin sesitivity

FIGURE 5: BDFI approach to study P. cocos and its active compounds for diabetes.

that $P . \operatorname{cocos}$ and triterpenes have the activities of insulin sensitizer but not releaser.

PPAR- $\gamma$ plays a role in glucose metabolism and is a target of insulin sensitizing drugs. Therefore, we further examined whether the crude extract of $P$. cocos and triterpenes regulated PPAR- $\gamma$ activation. As expected, rosiglitazone, a PPAR- $\gamma$ agonist, activated PPAR- $\gamma$ (Figure 4). However, the crude extract of $P$. cocos, dehydrotrametenolic acid, and pachymic acid did not activate PPAR- $\gamma$ (Figure 4). All the data suggest that $P$. cocos and triterpenes reduce blood glucose level via an enhancement of insulin sensitivity independent of PPAR- $\gamma$ (Figure 5).

\section{Discussion}

$P$. cocos is an edible fungus and is often used in various Chinese herbal medicines [13]. One of its claimed benefits is to treat diabetes; however, the actual anti-diabetic effects, active constituent(s), and mode of action of $P$. cocos have not been extensively studied. Here, we demonstrated, for the first time, that $P$. cocos shows an anti-diabetic effect in $\mathrm{db} / \mathrm{db}$ mice as evidenced by its glucose-lowering activity (Figure 2). Moreover, we showed, for the first time, that dehydrotumulosic acid is one of the most effective compounds present in the crude extract of $P$. cocos (Figure 2). Dehydrotrametenolic acid and, probably, pachymic acid were reported to be active compounds of $P$. cocos for glucose reduction in $\mathrm{db} / \mathrm{db}$ mice
[20]. Our data confirmed that dehydrotrametenolic acid and pachymic acid have lower glucose-reducing activities than dehydrotumulosic acid (Figure $2(\mathrm{~d})$ ). The anti-diabetic mechanism of $P$. cocos and its triterpenes are likely due to their abilities to sensitize insulin-mediated glucose uptake (Figure 3) [27]. Therefore, our findings provide insights into the mechanism of action of $P$. cocos and its active triterpenes against diabetes.

Lanostane-type triterpenes, pachymic acid, dehydrotrametenolic acid, polyporenic acid $\mathrm{C}$, dehydropachymic acid and 3-O-acetyl-16 $\alpha$-hydroxy-dehydrotrametenolic acid, and 3,4-secolanostane-type triterpenes, poricoic acid A, B, and $\mathrm{D}$ were identified from $P$. cocos [20]. Among these compounds, dehydrotrametenolic acid at $110 \mathrm{mg} / \mathrm{kg}$ body weight/day was confirmed as an active triterpene against hyperglycemia in $\mathrm{db} / \mathrm{db}$ mice. In this paper, dehydrotumulosic acid was identified from $P$. cocos crude extract using a bioactivity-directed fractionation and isolation strategy (Figures 1 and 2). However, dehydrotrametenolic acid (in chloroform sub-fraction 3 ) and pachymic acid (in chloroform sub-fraction 2) were not present in the active chloroform sub-fractions 4 and 6 of $P$. cocos. Two reasons may contribute to this contradiction. First, dehydrotrametenolic acid and pachymic acid have lower bioactivities than dehydrotumulosic acid. Second, we found that the percentages of dehydrotumulosic acid, dehydrotrametenolic acid, and pachymic acid in $P$. cocos crude extract were $0.012 \%$, 
$0.0022 \%$ and $0.053 \%$, respectively. The animal experiments in $\mathrm{db} / \mathrm{db}$ mice showed that dehydrotumulosic acid and dehydrotrametenolic acid had higher anti-hyperglycemic activity than pachymic acid (Figure $2(\mathrm{~d})$ ). Our data suggest that triterpenes are the primary compounds present in the crude extract of $P$. cocos despite their bioactivities with different degrees (Figures 1(a) and 2). Moreover, structures of these 3 compounds were confirmed by a comparison of the NMR, MS, and UV spectroscopy data obtained with published data from [21-23].

Crude extract of $P$. cocos and its triterpenes could reduce blood glucose via increased insulin sensitivity, as evidenced by STZ-treated mice (Figure 3). It is possible that the increase in insulin sensitivity could be partially achieved by modification/activation of the PPAR- $\gamma$ pathway. In fact, our data showed that $P$. cocos and its triterpenes did not activate PPAR- $\gamma$, a target of rosiglitazone (Figure 4). The mechanism by which crude extract of $P$. cocos and its triterpenes exert insulin sensitizer activity is PPAR- $\gamma$ independent. Biguanides (e.g., metformin) are another type of insulin sensitizer, which activate $5^{\prime}$ AMP-activated protein kinase. Whether or not $P$. cocos and its triterpenes affect $5^{\prime}$ AMP-activated protein kinase needs to be further studied [28].

Overall, db/db mice and STZ-treated mice experiments showed that the crude extract and triterpenes dehydrotumulosic acid and dehydrotrametenolic acid of $P$. cocos significantly enhanced the insulin sensitivity and, consequently, lowered the blood glucose level in a diabetic mouse model. This enhancement is independent of PPAR- $\gamma$ activation.

\section{Acknowledgments}

The authors thank Dr. Shih-Chang Chien for valuable suggestions and Dr. Krister Bamberg (Astra Zeneca, Mölndal, Sweden) for his plasmids. They also thank the ABRC Metabolomic Core Facility for technical assistance and Ms. Miranda Loney for revision of this paper. This research was supported by Academia Sinica and DPIAB Grant (099S0030093-AA).

\section{References}

[1] J. P. Boyle, M. M. Engelgau, T. J. Thompson et al., "Estimating prevalence of type 1 and type 2 diabetes in a population of African Americans with diabetes mellitus," American Journal of Epidemiology, vol. 149, no. 1, pp. 55-63, 1999.

[2] A. S. Attele, Y.-P. Zhou, J.-T. Xie et al., "Antidiabetic effects of Panax ginseng berry extract and the identification of an effective component," Diabetes, vol. 51, no. 6, pp. 1851-1858, 2002.

[3] M. Laakso, "Insulin resistance and its impact on the approach to therapy of type 2 diabetes," International Journal of Clinical Practice, Supplement, no. 121, pp. 8-12, 2001.

[4] T. H.-W. Huang, B. P. Kota, V. Razmovski, and B. D. Roufogalis, "Herbal or natural medicines as modulators of peroxisome proliferator-activated receptors and related nuclear receptors for therapy of metabolic syndrome," Basic and Clinical Pharmacology and Toxicology, vol. 96, no. 1, pp. 3-14, 2005.
[5] A. Y. Y. Cheng and I. G. Fantus, "Oral antihyperglycemic therapy for type 2 diabetes mellitus," Canadian Medical Association Journal, vol. 172, no. 2, pp. 213-226, 2005.

[6] H. C. S. Howlett and C. J. Bailey, "A risk-benefit assessment of metformin in type 2 diabetes mellitus," Drug Safety, vol. 20, no. 6, pp. 489-503, 1999.

[7] J. Q. Purnell and C. Weyer, "Weight effect of current and experimental drugs for diabetes mellitus: from promotion to alleviation of obesity," Treatments in Endocrinology, vol. 2, no. 1, pp. 33-47, 2003.

[8] A. J. Krentz and C. J. Bailey, "Oral antidiabetic agents: current role in type 2 diabetes mellitus," Drugs, vol. 65, no. 3, pp. 385$411,2005$.

[9] G. Z. Bi Zhishu and L. Taihui, The Macrofungus Flora of China's Guangdong Province, The Chinese University Press, Hong Kong, 1997.

[10] E. Boa, Wild Edible Fungi: A Global Overview of Their Use and Importance to People, Food \& Agriculture Organization of the UN, 2004.

[11] S. Li, Compendium of Materia Medica: Bencao Gangmu Beijing, Foreign Languages Press, Beijing, China, 2003.

[12] P. M. Kirk, P. F. Cannon, D. W. Minter, and J. A. Stalpers, Dictionary of the Fungi, CABI Publishing, Wallingford, UK, 2008.

[13] W. Jia, W. Gaoz, and L. Tang, "Antidiabetic herbal drugs officially approved in China," Phytotherapy Research, vol. 17, no. 10, pp. 1127-1134, 2003.

[14] U. Lindequist, T. H. J. Niedermeyer, and W.-D. Jülich, "The pharmacological potential of mushrooms," Evidence-Based Complementary and Alternative Medicine, vol. 2, no. 3, pp. 285-299, 2005.

[15] W. L. Li, H. C. Zheng, J. Bukuru, and N. De Kimpe, "Natural medicines used in the traditional Chinese medical system for therapy of diabetes mellitus," Journal of Ethnopharmacology, vol. 92, no. 1, pp. 1-21, 2004.

[16] T. Tai, A. Akahori, and T. Shingu, "A lanostane triterpenoid from Poria cocos,” Phytochemistry, vol. 31, no. 7, pp. 25482549, 1992.

[17] H. P. Molitoris, "Mushrooms in medicine," Folia Microbiologica, vol. 39, no. 2, pp. 91-98, 1994.

[18] P. C.-K. Cheung, "Dietary fibre content and composition of some edible fungi determined by two methods of analysis," Journal of the Science of Food and Agriculture, vol. 73, no. 2, pp. 255-260, 1997.

[19] J. K. Zjawiony, "Biologically active compounds from Aphyllophorales (polypore) fungi," Journal of Natural Products, vol. 67, no. 2, pp. 300-310, 2004.

[20] M. Sato, T. Tai, Y. Nunoura, Y. Yajima, S. Kawashima, and K. Tanaka, "Dehydrotrametenolic acid induces preadipocyte differentiation and sensitizes animal models of noninsulindependent diabetes mellitus to insulin," Biological \& Pharmaceutical Bulletin, vol. 25, no. 1, pp. 81-86, 2002.

[21] L. Chen, D. Wang, J. Wu, B. Yu, and D. Zhu, "Identification of multiple constituents in the traditional Chinese medicine formula GuiZhiFuLing-Wan by HPLC-DAD-MS/MS," Journal of Pharmaceutical and Biomedical Analysis, vol. 49, no. 2, pp. 267-275, 2009.

[22] T. Tai, A. Akahori, and T. Shingu, "Triterpenes of Poria cocos," Phytochemistry, vol. 32, no. 5, pp. 1239-1244, 1993.

[23] T. Tai, T. Shingu, T. Kikuchi, Y. Tezuka, and A. Akahori, "Triterpenes from the surface layer of Poria cocos," Phytochemistry, vol. 39, no. 5, pp. 1165-1169, 1995. 
[24] T. Nakagawa, A. Tsuchida, Y. Itakura et al., "Brain-derived neurotrophic factor regulates glucose metabolism by modulating energy balance in diabetic mice," Diabetes, vol. 49, no. 3, pp. 436-444, 2000.

[25] Y.-J. Hsu, T.-H. Lee, C. L.-T. Chang, Y.-T. Huang, and W.-C. Yang, "Anti-hyperglycemic effects and mechanism of Bidens pilosa water extract," Journal of Ethnopharmacology, vol. 122, no. 2, pp. 379-383, 2009.

[26] S.-L. Chang, C. L.-T. Chang, P.-I. Huang, M.-H. Tao, and W.-C. Yang, "Role of Cybr, a cytohesin binder and regulator, in $\mathrm{CD}^{+}$T-cell function and host immunity," Molecular Immunology, vol. 46, no. 16, pp. 3218-3223, 2009.

[27] S. Samane, J. Noël, Z. Charrouf, H. Amarouch, and P. S. Haddad, "Insulin-sensitizing and anti-proliferative effects of Argania spinosa seed extracts," Evidence-Based Complementary and Alternative Medicine, vol. 3, no. 3, pp. 317-327, 2006.

[28] J.-T. Hwang, M.-S. Lee, H.-J. Kim et al., "Antiobesity effect of ginsenoside Rg3 involves the AMPK and PPAR- $\gamma$ signal pathways," Phytotherapy Research, vol. 23, no. 2, pp. 262-266, 2009. 


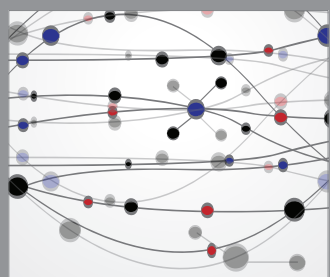

The Scientific World Journal
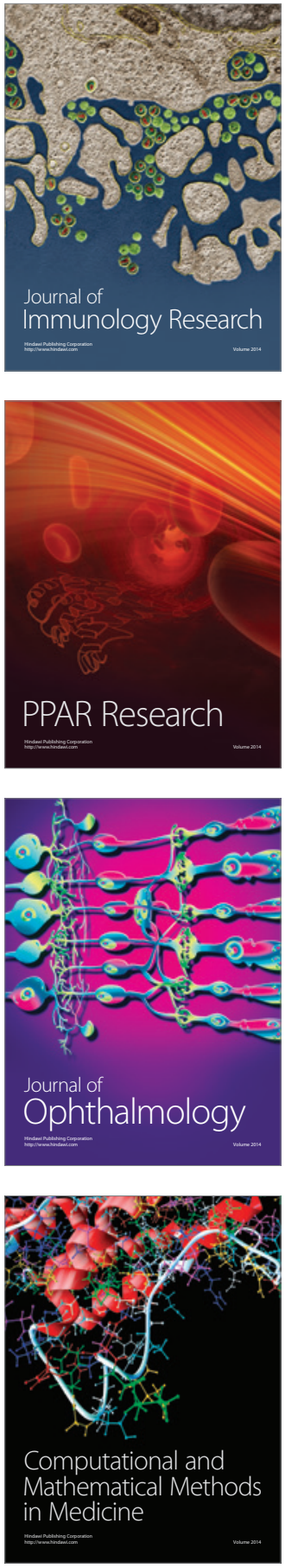

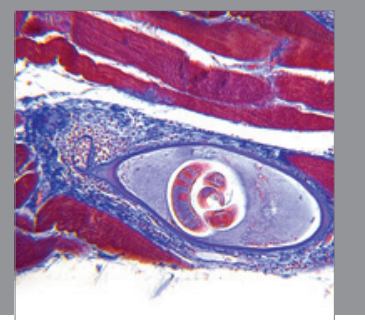

Gastroenterology

Research and Practice
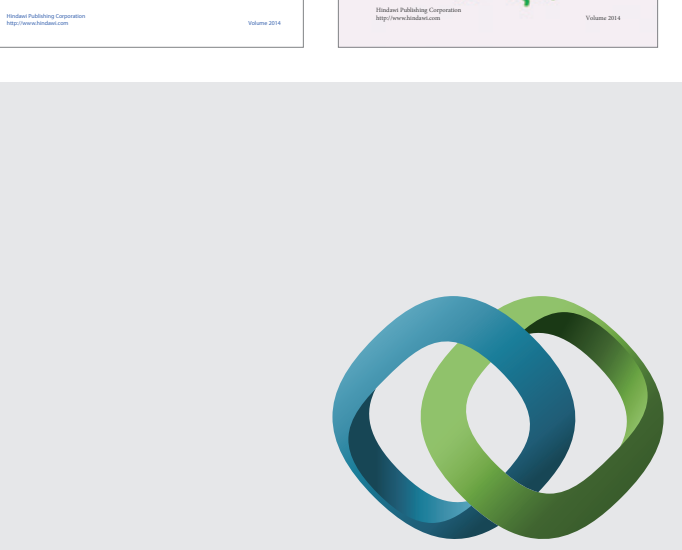

\section{Hindawi}

Submit your manuscripts at

http://www.hindawi.com
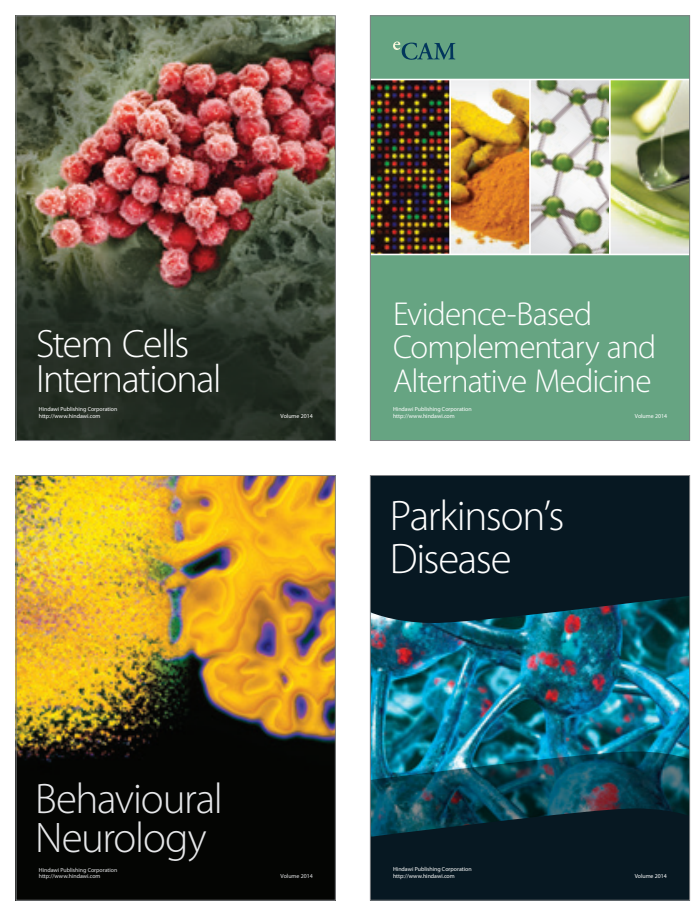

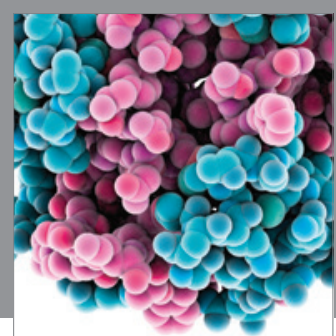

Journal of
Diabetes Research

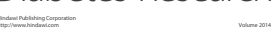

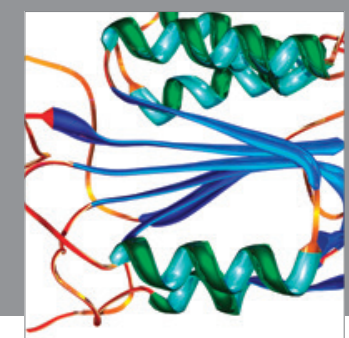

Disease Markers
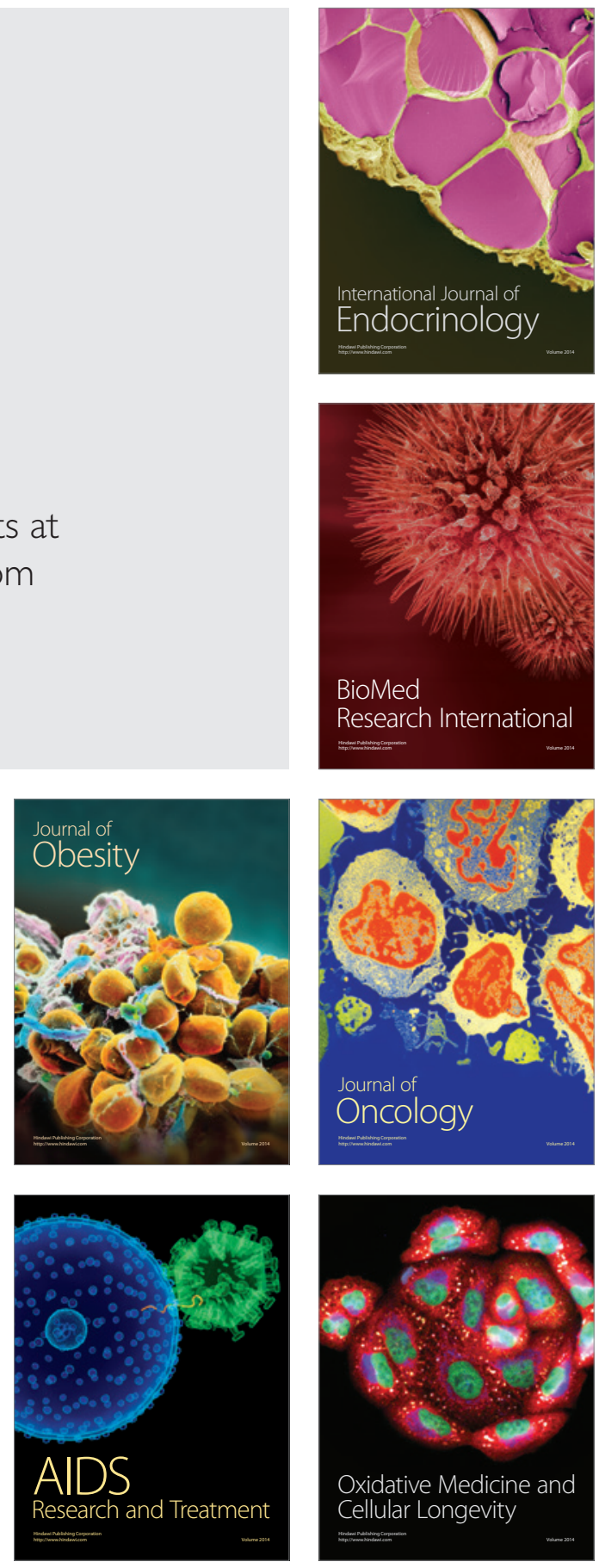